\title{
The Acute Calcific Prevertebral Tendinitis : Report of Two Cases
}

\author{
Dong-Eun Shin', Chang-Soo Ahn², Jung-Pil Choi' \\ 'Department of Orthopaedic Surgery, CHA Bundang Medical Center, \\ CHA University College of Medicine, Seongnam, Korea \\ ${ }^{2}$ Department of Orthopaedic Surgery, CHA Gumi Medical Center, CHA University College of Medicine, Gumi, Korea
}

\begin{abstract}
Acute calcific prevertebral tendinitis, which is also known as retropharyngeal calcific tendinitis and longus colli tendinitis, is an under-recognized cause of acute cervical pain produced by an inflammation of the longus colli muscle. The typical characteristics of this entity are calcifications at the superior insertion of the longus colli tendons at the $\mathrm{C} 1-\mathrm{C} 2$ level and fluid collection in the retropharyngeal space. The differential diagnosis includes a retropharyngeal abscess, infectious spondylitis or traumatic injury. Knowledge of the clinical and imaging findings can prevent a misdiagnosis and inappropriate attempts at surgical drainage.
\end{abstract}

Key Words: Longus colli, Retropharynx, Calcific tendinitis

\section{Introduction}

Acute calcific prevertebral tendinitis is an unusual benign condition caused by calcium hydroxyapatite deposition in the longus colli tendon and inflammation of the longus colli muscle. The clinical presentation is often non-specific and is characterized by spontaneous acute neck pain, dysphagia or odynophagia, sometimes accompanied by low-grade fever. Laboratory tests may demonstrate inflammatory signs, such as mild leukocytosis and slightly elevated erythrocyte sedimentation rate. The initial workup of patients is often performed to exclude more serious causes of acute neck pain, such as a traumatic injury, retropharyngeal abscess, meningitis and infectious spondylitis. This condition can easily be overlooked or misdiagnosed due to its rather non-specific presentation and rare occurrence [1]. A diagnosis is made easily by the recognition of its pathognomonic plain film and computed tomography (CT) appear- ance [2]. We report two patients with acute calcific prevertebral tendinitis.

\section{Case Reports}

\section{Case 1}

A 22-year-old female patient presented with a 2-day history of a painful stiff neck and odynophagia. There was no associated fever, recent illnesses or trauma history. The physical examinations revealed a reduced active range of motion in all directions, particularly on the left lateral side. There was no other radiating pain or neurological symptoms to the upper extremities. We checked for mild tenderness on the left and posterior neck area. The oropharyngeal examination was normal, with no associated cervical lymphadenopathy. The initial standard X-rays of the cervical spine revealed diffuse mild swelling on the retropharyngeal space and suspicious calcific density inferior to the $\mathrm{C} 1$ ante-

Received Oct 17, 2009; 1st Revised Dec 30, 2009; 2nd Revised Feb 3, 2010; Accepted Feb 3, 2010

Corresponding author: Chang-Soo Ahn, MD

Department of Orthopaedic Surgery, CHA Gumi Medical Center,

855 Hyeonggok-dong, Gumi 730-040, Korea

Tel : +82-54-450-9571, Fax : +82-31-708-3578, E-mail : shinde@cha.ac.kr 
rior arch. The follow-up X-rays after 1month showed considerably improved prevertebral swelling with retropharyngeal calcific tendinitis of the longus colli (Fig. 1).

Subsequent CT of the cervical spine confirmed that the curvilinear calcific density was inferior to the $\mathrm{C} 1$ anterior arch, and anterior to the $\mathrm{C} 2$ body with mild prevertebral soft tissue thickening (approximately $1 \mathrm{~cm}$ in maximal thickness) (Fig. 2). The magnetic resonance imaging (MRI) images revealed a hypointense nodular lesion inferior to the $\mathrm{C} 1$ anterior arch and anterior to the $\mathrm{C} 2$ body. Associated minimal prevertebral edema was also observed (Fig. 3).

After 7 days of admission, she had recovered after receiv-
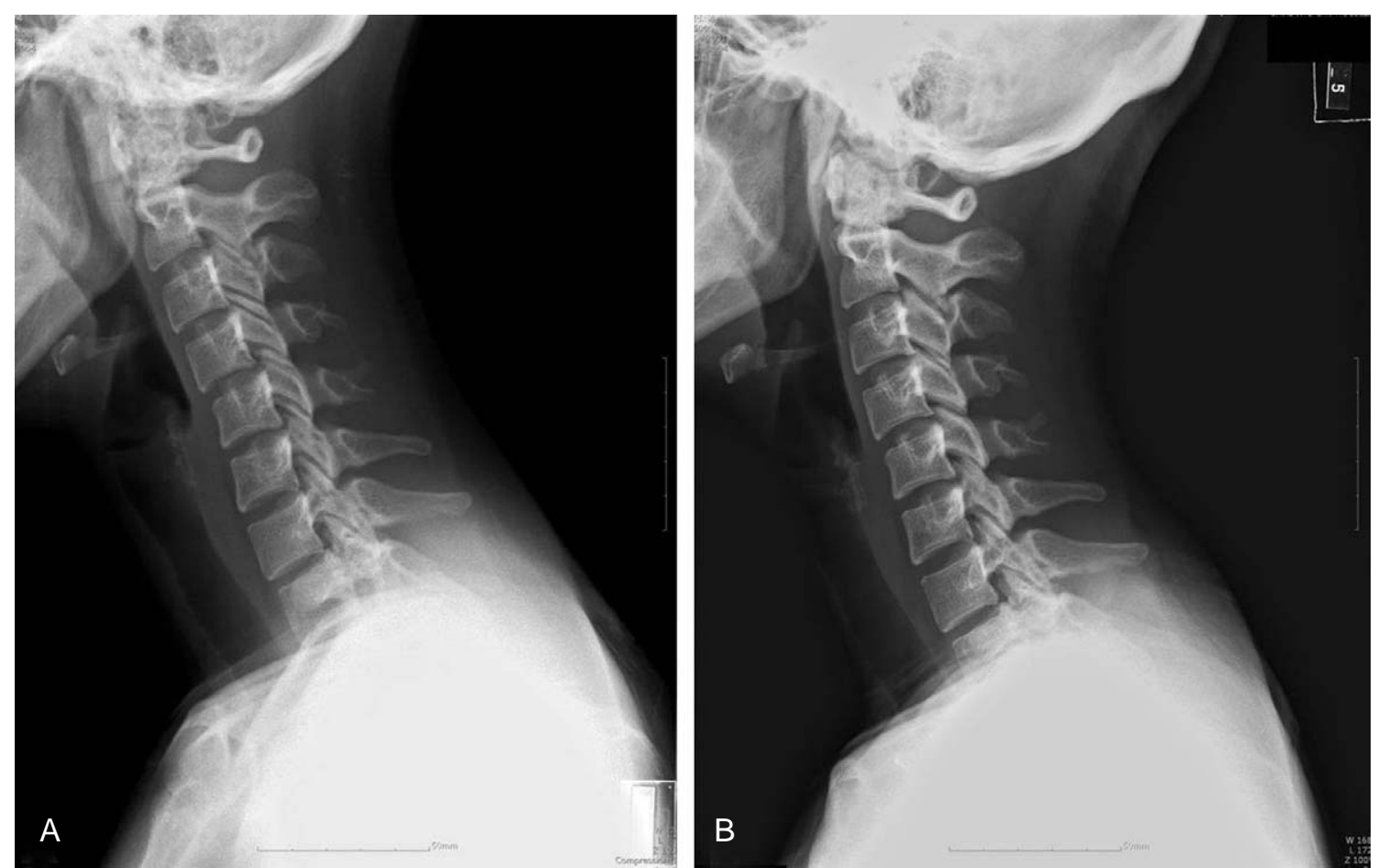

Fig. 1. Initial and follow up C-spine lateral view. Initial C-spine lateral view. Diffuse mild swelling on the retropharyngeal space. Suspicious calcific density inferior to the $\mathrm{C} 1$ anterior arch (A). Follow up C-spine lateral view. Much improved prevertebral swelling with retropharyngeal calcific tendinitis of longus colli (B).

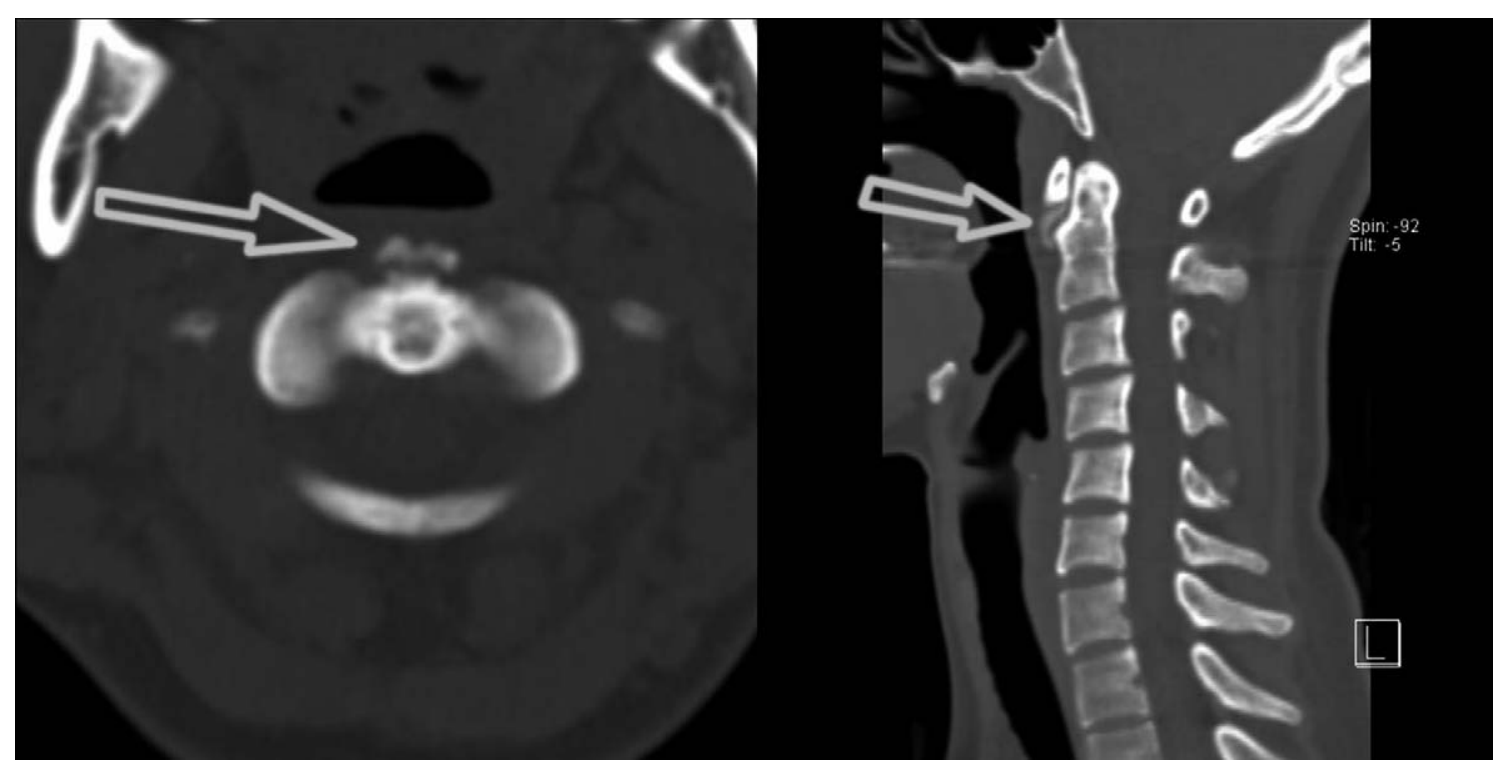

Fig. 2. Initial computed tomography. Curvilinear calcific density is observed inferior to the $\mathrm{C} 1$ anterior arch, and anterior to the $\mathrm{C} 2$ body with mild prevertebral soft tissue thickening (approximately $1 \mathrm{~cm}$ in maximal thickness). 
ing conservative treatment, including anti-inflammatory drugs and rest.

\section{Case 2}

A 51-year-old male patient presented with a 1 week history of painful neck stiffness. The pain was aggravated and the range of motion was severely limited. There was no history of fever, night sweats, recent illnesses or traumatic events. The physical examinations revealed a reduced active range of motion in flexion and extension due to pain. The patient complained of odynophagia but flexible nasopharyngolaryngoscopy by an otolaryngologist showed no other

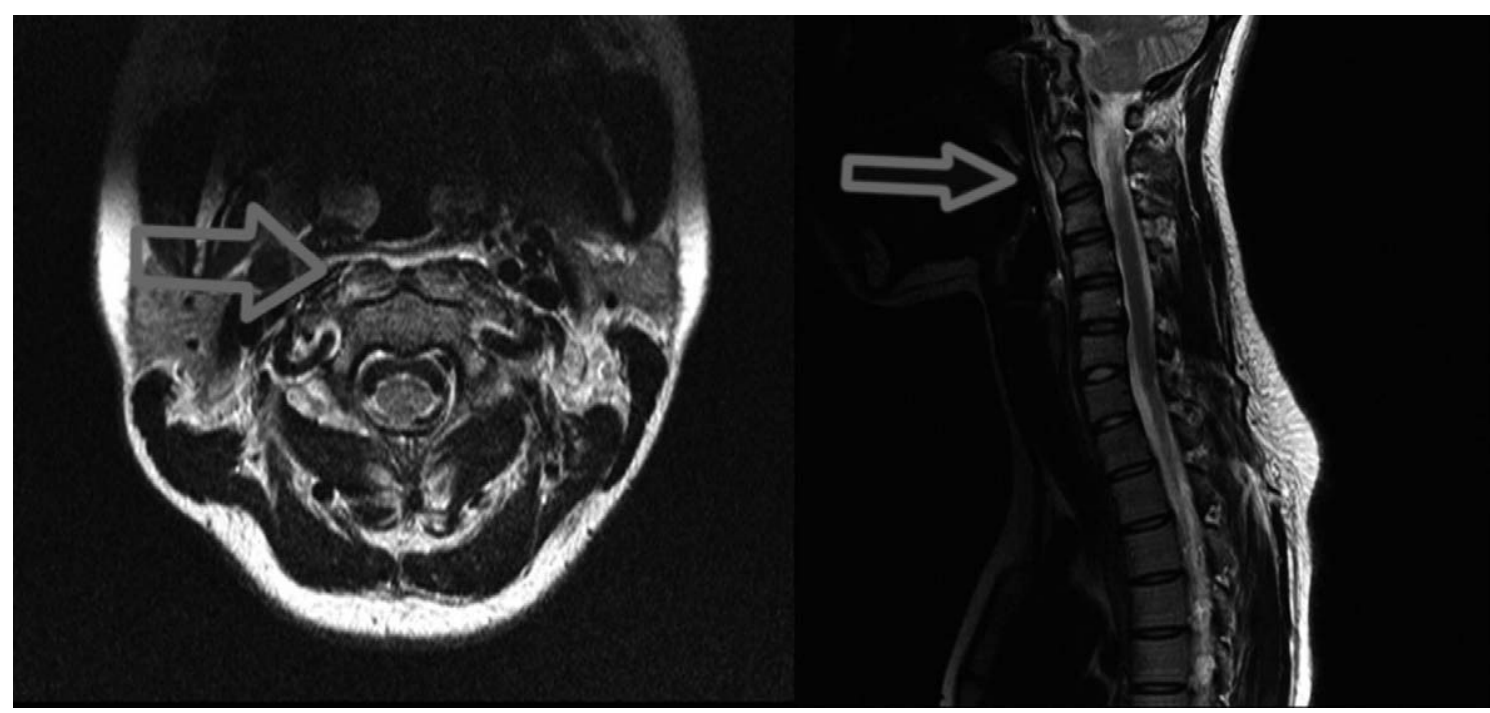

Fig. 3. Initial magnetic resonance imaging (MRI). Initial C-spine MRI T2-weighted axial and sagittal view. Hypointense nodular lesion is observed inferior to the $\mathrm{C} 1$ anterior arch and anterior to the $\mathrm{C} 2$ body (arrows). Associated minimal prevertebral edema is observed in retropharyngeal space.
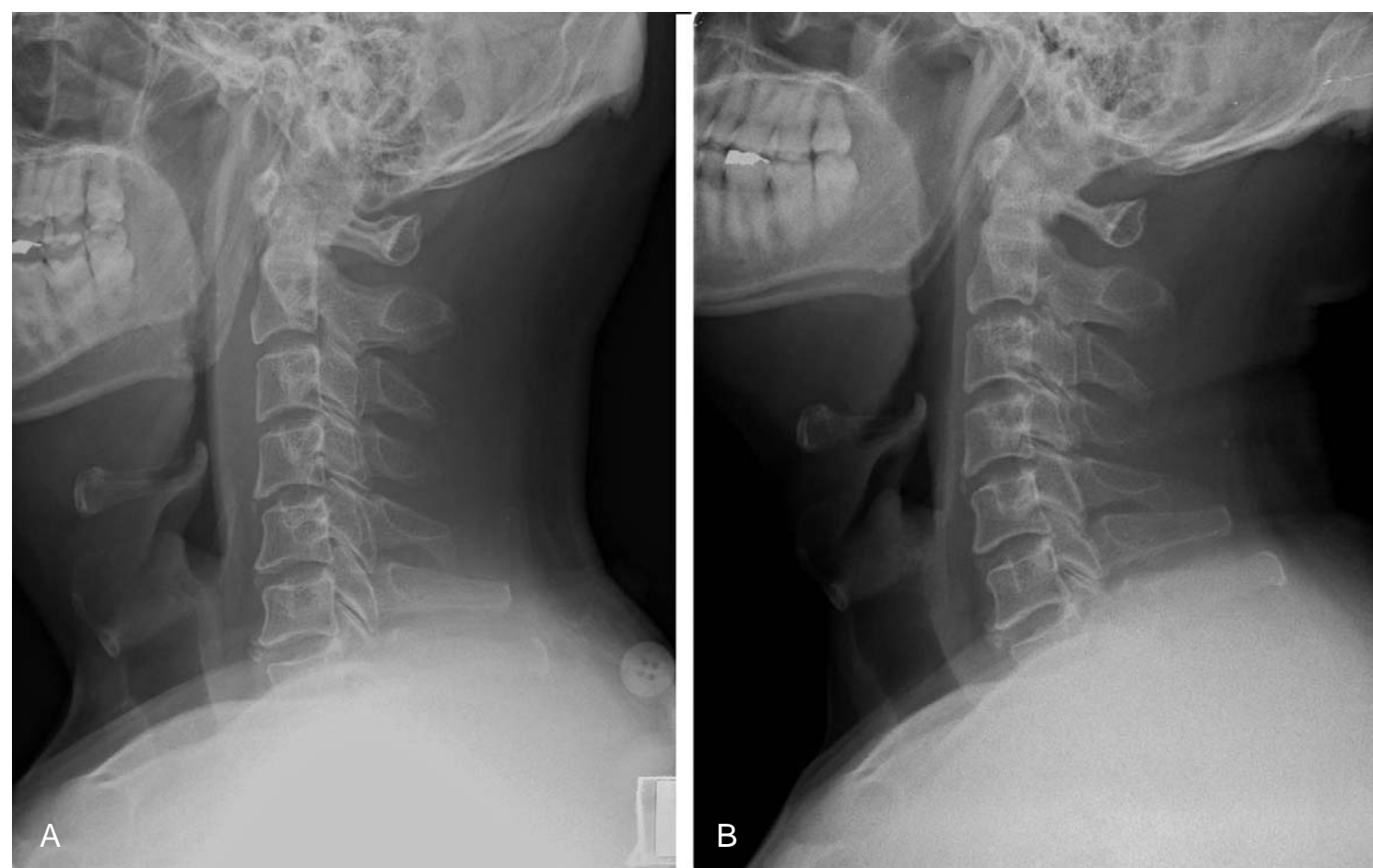

Fig. 4. Initial and follow up C-spine lateral view. Initial C-spine lateral view. Diffuse swelling on the retropharyngeal space with compression of the airway. Suspicious calcific density inferior to the $\mathrm{C} 1$ anterior arch (A). Follow up Cspine lateral view. No evidence of calcific density or airway compression (B). 
abnormal findings in the oropharyngeal space. The laboratory tests revealed mild leukocytosis. The initial C-spine lateral X-rays revealed diffuse swelling on the retropharyngeal space with compression of the airway and a suspicious calcific density inferior to the $\mathrm{C} 1$ anterior arch. The followup X-rays after 1 month showed no evidence of calcific density or airway compression (Fig. 4).

An MRI examination was subsequently performed, which showed a focal dark signal intensity nodular lesion inferior to the anterior arch of $\mathrm{C} 1$ without any evidence of contrast enhancement, and associated compression of the airway was noted (Fig. 5).

The patient was not admitted and received conservative treatment, including anti-inflammatory drugs and rest. The symptoms resolved after 1 month and the follow-up radiograph performed approximately 1 month later demonstrated complete resolution.

\section{Discussion}

Retropharyngeal calcific tendinitis, which is also known as acute calcific prevertebral tendinitis, is a clinical syndrome that was first described by Hartley [3] in 1964, and was demonstrated by Ring et al. [4] in 1994 to be secondary to calcium hydroxyapatite deposition in the longus colli muscle. This muscle is a paired neck flexor muscle that contains the prevertebral space. The longus colli muscle consists of 3 portions: superior oblique, inferior oblique and vertical. Classically, the calcification affects the superior oblique portion of the longus colli muscle at the $\mathrm{C} 1-\mathrm{C} 2$ level [5]. Acute prevetebral calcific tendinitis can go unrecognized and be misdiagnosed due to its rare occurrence. Acute prevetebral calcific tendinitis is a form of calcium hydroxyapatite deposition disease that specifically involves the longus colli muscle and tendons. This is an uncommon location for a disease that more typically involves the large joints, such as the hips or shoulders. The etiology of calcium hydroxyapatite crystal deposition is unclear, and there are some suggestions that repetitive trauma, recent injury, tissue necrosis, or ischemia may play a role. It is believed that once the calcium crystals have deposited within the longus colli muscle, they invoke a painful foreign body-like inflammatory response [6]. The condition affects adults within a reported age range of 21 to 81 years, with the greatest distribution between 30 and 60 years. The symptoms are secondary to inflammation of the longus colli and include an acute to subacute onset of neck pain, dysphagia, odynophagia, and low-grade fever. The dysphagia is due to the close proximity of the retropharyngeal space to the adjacent pharyngeal constrictors [7].

The principal radiographic findings of retropharyngeal calcific tendinitis include prevertebral soft tissue swelling, and amorphous calcification anterior to C1-C2. The diffuse prevertebral soft tissue thickening typically extends from $\mathrm{C} 1$ to $\mathrm{C} 4$, even though it can extend inferiorly to C6 [8]. The soft tissue thickening represents either discrete effusion

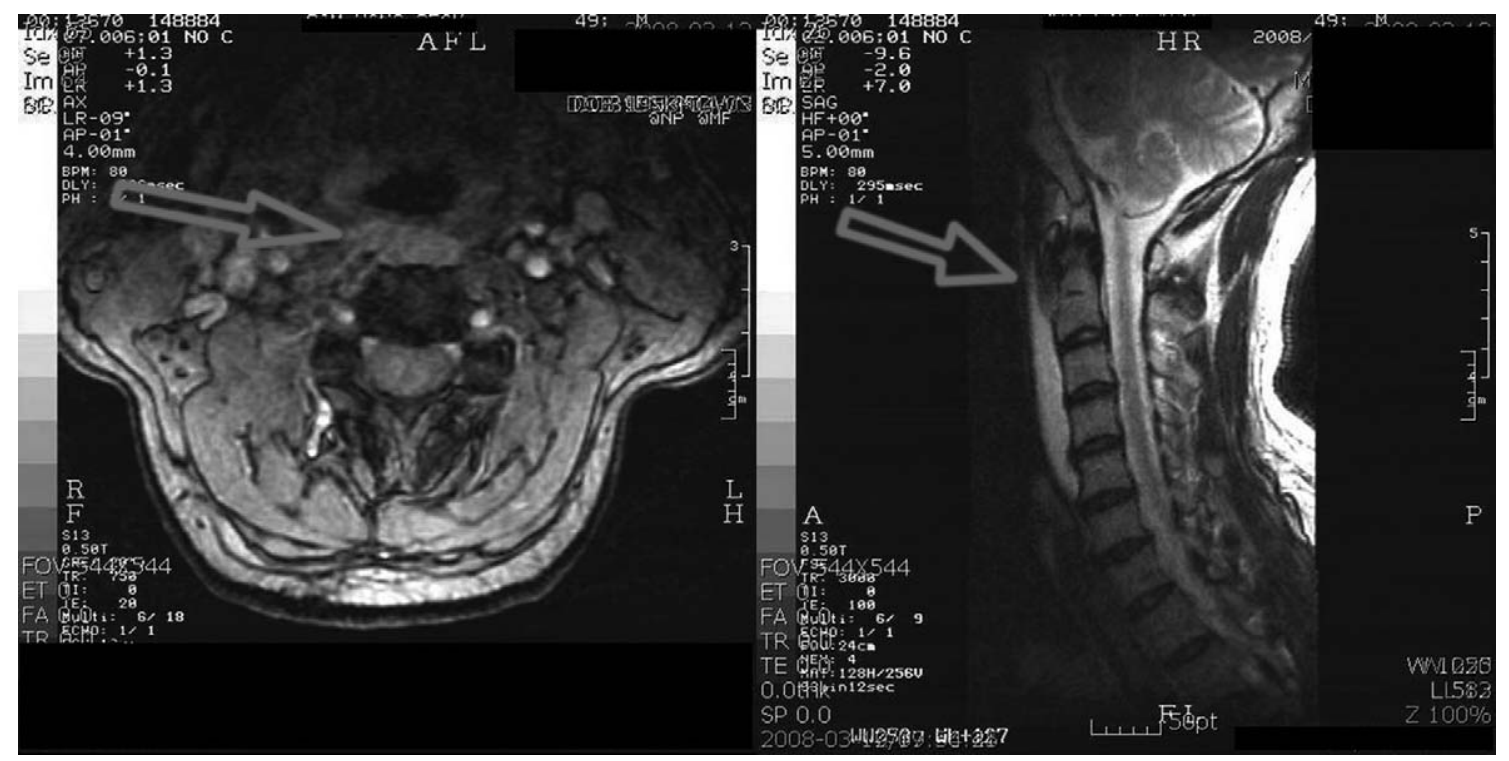

Fig. 5. Initial magnetic resonance imaging (MRI). Initial C-spine MRI T2-weighted axial and sagittal view. Focal signal intensity nodular lesion is noted inferior to the anterior arch of $\mathrm{C} 1$ and $\mathrm{C} 2$ body (arrows). There is no evidence of contrast enhancement. Associated compression of the airway can be seen. 
or diffuse edema, which can be differentiated on CT or MRI. The lack of enhancement surrounding the effusion can be helpful in differentiating a reactive effusion from an abscess. The CT scan can help identify calcific deposits, which can often appear faint on plain film. MRI is not typically necessary for this diagnosis but can sometimes demonstrate marrow edema in the adjacent vertebrae [9].

Acute prevetebral calcific tendinitis is a self-limiting condition that resolves spontaneously after 1-2 weeks. If the calcific tendonitis is not treated, it is self-limiting and resolves within several weeks of the onset of symptoms, even though it is quite painful and debilitating to the patient during that time. Some forms of conservative treatment, such as a short course of non-steroidal anti-inflammatory medication and the avoidance of aggravating neck movements, can help alleviate the symptoms. Familiarity with this rare entity and its pathognomonic imaging findings can prevent incorrect medical therapy and invasive attempts at surgical drainage. Furthermore, by being aware of this condition, one may save the patient from unnecessary diagnostic workup, undue anxiety and delays in hospital discharge. If a CT neck report indicates nonspecific fluid collection in the retropharyngeal space, an astute clinician familiar with this condition may wish to review the films with the radiologist to determine if the $\mathrm{C} 1-\mathrm{C} 2$ calcification is present.

\section{REFERENCES}

1. Bladt O, Vanhoenacker R, Bevernage C, Van Orshoven M, Van Hoe L, D'Haenens P. Acute calcific prevertebral tendinitis. JBR-BTR 2008;91:158-9.

2. Chung T, Rebello R, Gooden EA. Retropharyngeal calcific tendinitis: case report and review of literature. Emerg Radiol 2005; $11: 375-80$.

3. Hartley J. Acute cervical pain associated with retropharyngeal calcium deposit: a case report. J Bone Joint Surg Am 1964;46:1753-4.

4. Ring D, Vaccaro AR, Scuderi G, Pathria MN, Garfin SR. Acute calcific retropharyngeal tendinitis: clinical presentation and pathological characterization. J Bone Joint Surg Am 1994;76:1636-42.

5. Razon RV, Nasir A, Wu GS, Soliman M, Trilling J. Retropharyngeal calcific tendonitis: report of two cases. J Am Board Fam Med 2009;22:84-8.

6. De Maeseneer M, Vreugde S, Laureys S, Sartoris DJ, De Ridder F, Osteaux M. Calcific tendinitis of the longus colli muscle. Head Neck 1997;19:545-8.

7. Omezzine SJ, Hafsa C, Lahmar I, Driss N, Hamza H. Calcific tendinitis of the longus colli: diagnosis by CT. Joint Bone Spine 2008;75:90-1.

8. Fahlgren H. Retropharyngeal tendinitis: three probable cases with an unusually low epicentre. Cephalalgia 1988;8:105-10.

9. Mihmanli I, Karaarslan E, Kanberoglu K. Inflammation of vertebral bone associated with acute calcific tendinitis of the longus colli muscle. Neuroradiology 2001;43:1098101. 